Modeling, Identification and Control, Vol. 33, No. 3, 2012, pp. 99-109, ISSN 1890-1328

\title{
Numerical and Experimental Study of Friction Loss in Hydrostatic Motor
}

\author{
Rasmus M. Sørensen ${ }^{1}$ Michael R. Hansen ${ }^{2}$ Ole $\varnothing$. Mouritsen $^{3}$ \\ ${ }^{1}$ Liftra Aps \&5 Department of Mechanical and Manufacturing Engineering, Aalborg University, DK-9220 Aalborg \\ East, Denmark. E-mail: rms@liftra.dk \\ ${ }^{2}$ Department of Engineering, University of Agder, N-4876 Grimstad, Norway. E-mail: michael.r.hansen@uia.no \\ ${ }^{3}$ Department of Mechanical and Manufacturing Engineering, Aalborg University, DK-9220 Aalborg East, Denmark. \\ E-mail: oom@aau.dk
}

\begin{abstract}
This paper presents a numerical and experimental study of the losses in a hydrostatic motor principle. The motor is designed so that the structural deflections and lubricating regimes between moving surfaces and, subsequently, the leakage and friction losses, can be controlled during operation. This is done by means of additional pressure volumes that influence the stator deflection. These pressures are referred to as compensation pressures and the main emphasis is on friction or torque loss modeling of the motor as a function of the compensation pressures and the high and low pressures related to the load torque. The torque loss modeling is identified as a Stribeck curve which depends on gap height. The asperity friction is decreasing exponentially with an increase in gap height. The parameters of the torque loss model are based on prototype measurements that include the structural deflections of the lubricating gap faces.
\end{abstract}

Keywords: Hydrostatic motor, friction loss, mixed lubrication, experimental verification

\section{Introduction}

The performance of hydrostatic displacement machines is strongly related to their efficiencies. The design and manufacturing tolerances are a trade-off between volumetric and hydromechanical loss. The general trend is that if the volumetric loss goes down the hydromechanical loss goes up, and vice versa, because of their opposite nature. Except for the division into volumetric and hydromechanical losses there exist no unified modeling techniques that describe the losses in hydrostatic machines in detail (Ivantysyn and Ivantysynova, 2003). Especially, the hydromechanical loss is difficult to model in a simple way since it depends on a wide variety of friction losses. They include laminar and turbulent flow induced pressure drops in the displaced fluid as well as a combination of friction caused by boundary layer, mixed layer and elastohydrodynamic lubrication between moving parts. These phenomena depend on the operating conditions as well as the topology and geometry of the hydrostatic machine.

In general, the complex behavior prevents model based prediction of the performance and therefore design of hydrostatic machines rely heavily on fine machining tolerances and excessive testing of volumetric and hydromechanical losses for the entire operating range, see (Ivantysyn and Ivantysynova, 2003), (Ivantysynova, 2003) and (Murrenhoff et al., 2008).

In this work the hydromechanical efficiency is examined for a new motor concept. The concept has been presented in earlier work (Sørensen et al., 2011). The most important characteristics of the motor principle are a high specific displacement (displacement per motor volume), a minimum of manufacturing tolerance 
dependencies and the ability to adjust structural deflections of leakage gaps by applying so-called compensation pressures via designated pressure pockets.

Work has already been done on the leakage characteristics of the motor principle with regard to compensation pressure (Sørensen et al., 2012). In (Sørensen et al., 2012) the conclusion is that the volumetric efficiency of the motor principle is highly influenced by the gap flow across the large end faces of the rotor and the housing, and that an increase in these gap heights can be prevented by the use of compensation pressures. In fact, both theoretical and experimental studies clearly revealed that any succesful application of the motor depends on the use of the compensation pressure to reduce leakage flow. Obviously, this compensation must be applied carefully to avoid excessive friction losses.

Therefore, this paper is on the investigation of the torque losses of the motor principle. The main contributions are:

- The set up of an experimentally verified model to describe the surface gap between the rotor and the inner housing

- The set up of an experimentally verified model to describe the torque loss between the rotor and the inner housing

- The development of an understanding of the interaction between the rotor and the inner housing.

\section{Motor Principle}

The hydraulic motor principle investigated in this paper is shown in figure 1 . The stops and the rotor form six chambers that are further divided by one or two vanes. However, it can be designed with different number of chambers and vanes as a trade off between specific displacement and complexity. The rotor is subjected to a torque because of pressure difference across the vanes. The vanes are moved in radial direction when passing a stop. This radial movement of the vanes is facilitated by means of alternating high and low hydraulic pressures in pockets in the housing. These pressures are referred to as vane actuation pressures $\left(p_{v}\right)$ and they are reversed together with the rotational direction of the rotor. In figure 1 vane actuation pressures corresponding to a clockwise rotation are shown. The vane actuation pressures are symmetrical on both side of the rotor to avoid an axial force imbalance on the rotor. When not passing a stop the vanes are forced against the stator which seals with the vane tip. To avoid high demands on tolerances on the dominant radial dimension the stops are designed as small pistons that are forced against the rotor by a pressure.

The two large areas between the rotor and the inner housing (the face on the middle view of figure 1) strongly influence the efficiency of the motor. These areas have, as all the gaps between moving parts, double

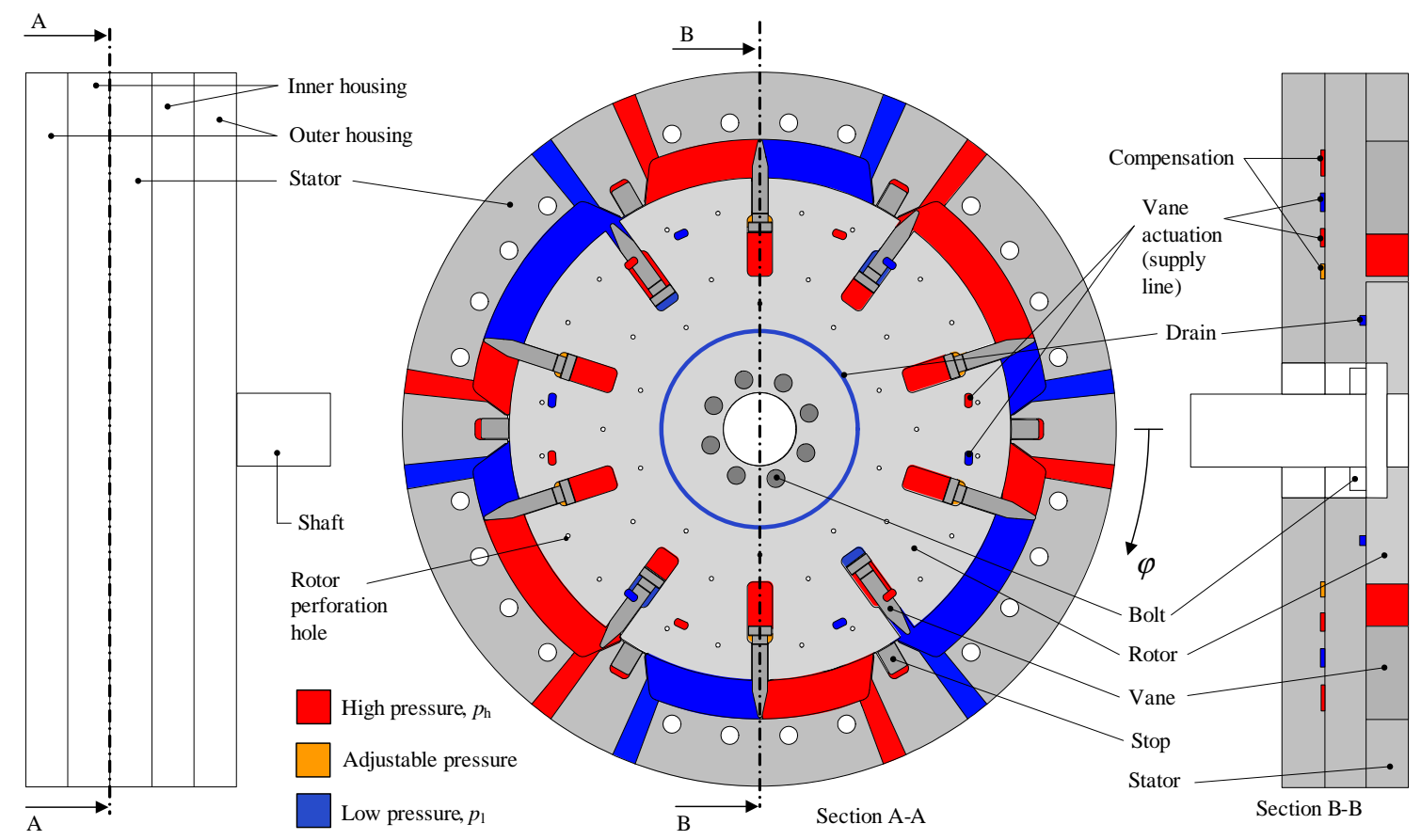

Figure 1: The motor principle. 
contradicting functionality, namely sealing and lubrication. If the performance criteria of either of these functionalities are not given proper attention the motor will not be useful for practical applications. The main challenge is to maintain the performance with respect to both performance criteria over a wider range of operating conditions. The pressure distribution in these gaps is creating a significant force at the inner housings because of the large area from the outer radius of the chambers to the drain connections located close to the centre of the rotor. These forces are causing a deflection of the inner housings which must be minimized to maximize the volumetric efficiency of the motor. To counteract these deflections, the motor has compensation pressure volumes between the inner and outer housings (see location and geometry in figure 1 , 2 and 3$)$.

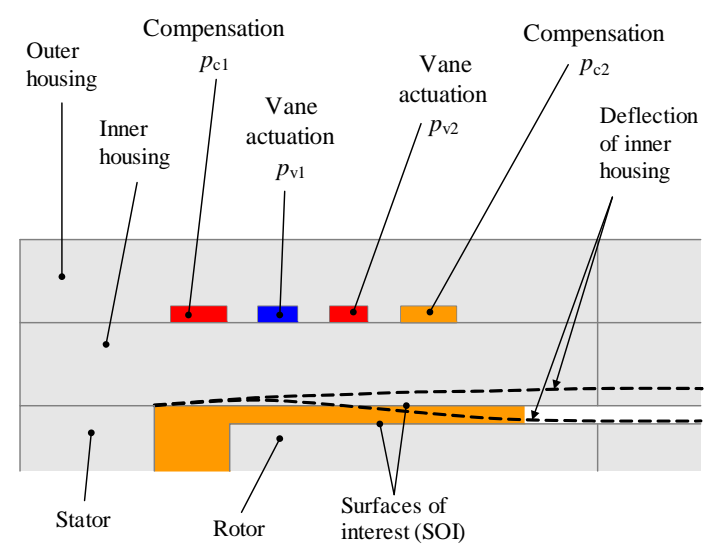

Figure 2: A section view of the motor principle with potential deflections of an inner housing illustrated by the dotted lines.

Besides the pressure distribution in the gap and the compensation pressures, the pressures in the supply lines to the vane actuation also influence the deflections of the inner housings. Therefore the deflection of the inner housings can potentially be in two directions as illustrated by the dotted lines in figure 2 . The deflection behavior depends on the area and pressure magnitude of the individual volumes illustrated in figure 2. The layout of the vane actuation supply lines and compensation pressure volumes is restricted by the available space outside the sealings and oil channels. The layout of these volumes is illustrated in figure 3 without oil inlet channels. The volumes are sealed by o-rings.

The surfaces of interest (SOI) in this paper are shown in figure 2 with potential gaps illustrated by the dotted lines. The pressures in the vane actuation supply lines follow the low and high pressure levels of the motor.

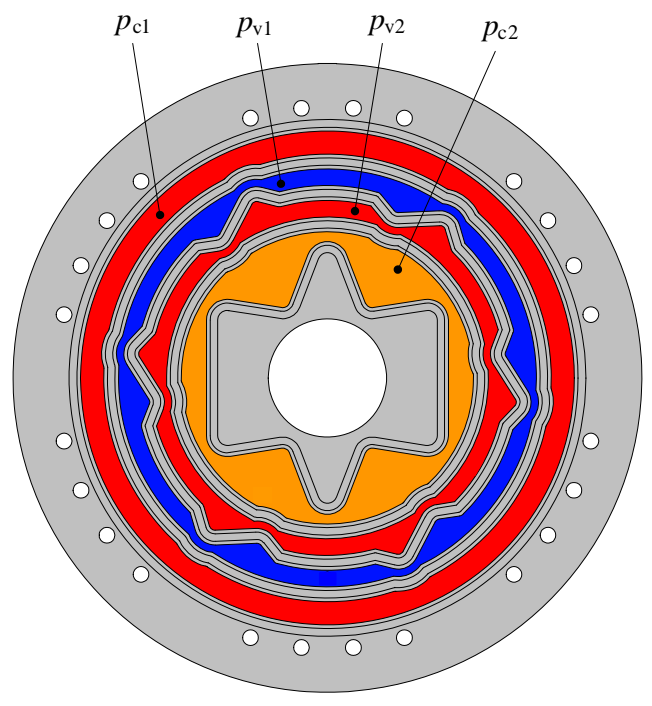

Figure 3: The geometry of the compensation and vane actuation supply pressure volumes, which are between the inner and outer housing.

One is connected to the low pressure part and the other to the high pressure part, depending on the rotational direction.

The velocity of the motor and the oil viscosity are held constant in this work, because the torque loss due to these phenomena are not remarkably different as compared to existing hydrostatic motor principles. The remaining friction losses that are singled out for attention are:

- the friction force of the boundary lubrication between stops and rotor

- the friction force of the boundary lubrication between vanes and stator

- the friction force of the boundary, mixed or elastohydrodynamic lubrication (EHL) between the end faces of the rotor and the inner housings

\section{Experimental Work}

The aim of the experimental work is to investigate the torque loss and the influence of the compensation pressures on the torque loss. In the experiments $p_{c 1}$ is equal to the high pressure, $p_{h}$, of the prototype and $p_{c 2}$ is adjusted to different pressure levels to examine the changing lubrication regime between the SOI.

\subsection{Experimental Test Setup}

To be able to determine the torque losses of the prototype motor the test setup has pressure transducers to 


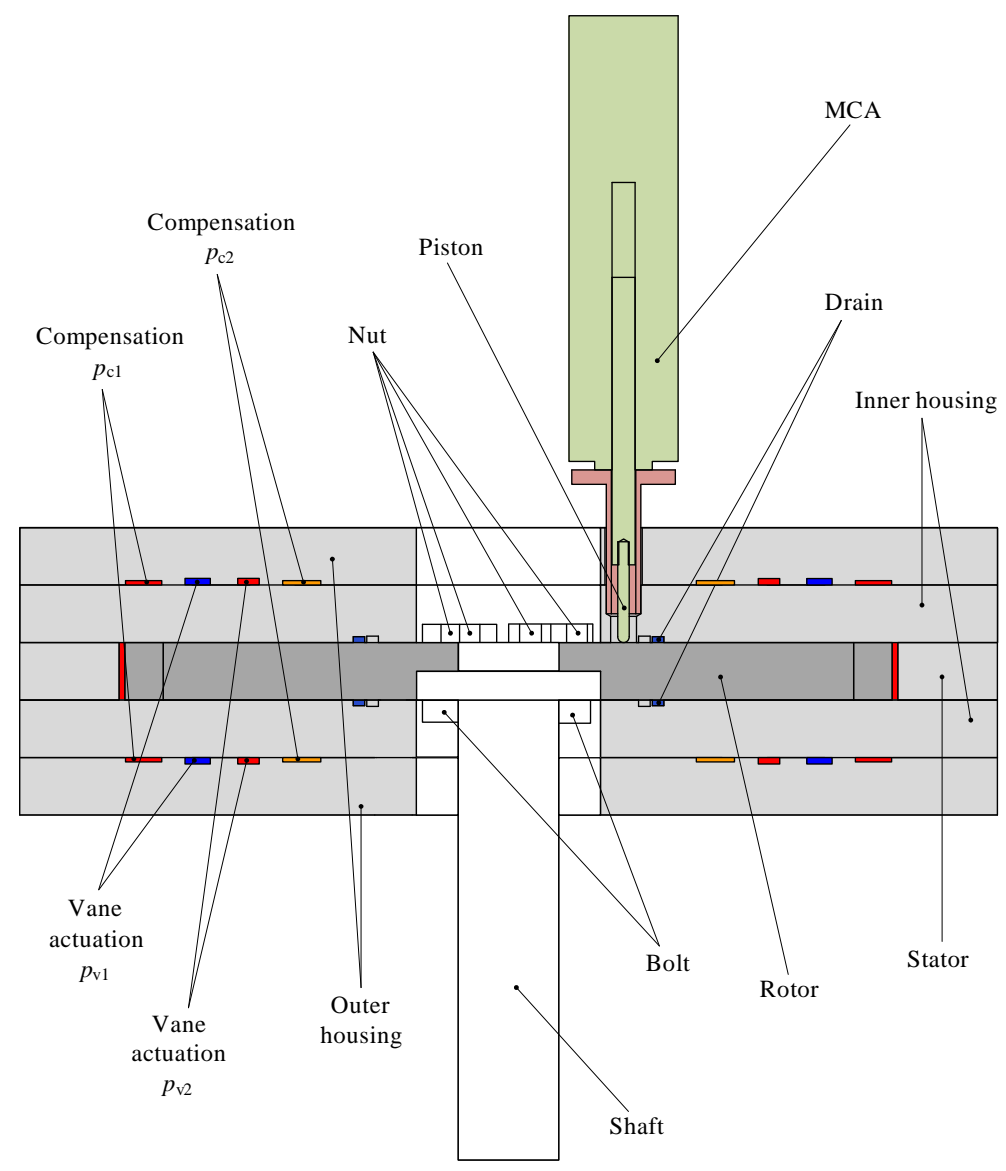

Figure 4: Section cut of the prototype motor. The moving coil actuator (MCA) is mounted on the inner housing and the piston is forced against the end face of the rotor during tests.

measure $p_{h}$ and $p_{l}$, see figure 1 , yielding the pressure difference, $\Delta p=p_{h}-p_{l}$, and a strain gauge transducer to give the output torque $T_{\text {out }}$. Further the test setup has flow transducers, a temperature transducer and an absolute encoder for motor velocity. The prototype motor is loaded by a powder brake, through a gearbox.

The structural deflections of the inner housing are measured with a moving coil actuator (MCA). The MCA is fitted with a $0.1 \mu \mathrm{m}$ encoder to measure the position of the MCA piston. The body is mounted on the inner housing of the prototype motor as illustrated in figure 4. The MCA is controlled in force mode and maintains a force of $1.7 \mathrm{~N}$ during the tests so that the piston always is in contact with the rotor. The builtin encoder of the MCA measures the position of the piston and therefore the relative distance between the inner housing and the rotor. The measurements are performed when the rotor rotates and therefore the MCA tip is sliding across the face of the rotor. The extracted gap heights are mean values during one motor revolution to minimize deviations due to e.g. surface roughness and variations in the thickness of the rotor.
The adjusted compensation pressure $p_{c 2}$ is restricted by a pressure reducing valve. During the tests, the pressure level is adjusted to $p_{c 2}=10,16,22,27 \mathrm{bar}$.

The data for the prototype motor and the motor conditions during the experimental tests, are listed in table 1.

\subsection{Experimental Results}

The total torque loss of the prototype motor is

$$
T_{\text {loss }}=T_{\text {th }}-T_{\text {out }}=\frac{\left(p_{h}-p_{l}\right) \cdot D}{2 \cdot \pi}-T_{\text {out }},
$$

where $D$ is the displacement. The measured $T_{l o s s}$ and the measured deflections of the inner housing $h_{M C A}$ are shown in figure 5. The trend in the measurement is clear. $T_{\text {loss }}$ is decreasing with the increase in $p_{h}$ until reaching a minimum $T_{\text {loss,min }}$, whereafter $T_{\text {loss }}$ is increasing almost linearly with $p_{h}$. $T_{\text {loss,min }}$ is changing for the different $p_{c 2}$ values and for $p_{c 2}=27$ bar the minimum is not inside the pressure range of the tests. The hypothesis put forward here is that before $T_{l o s s, m i n}$ 
Table 1: The prototype specifications and the operating conditions during the tests.

\begin{tabular}{ll}
\hline Outer motor diameter & $d=340 \mathrm{~mm}$ \\
\hline Outer motor height & $l=100 \mathrm{~mm}$ \\
\hline Height of rotor & $s=20 \mathrm{~mm}$ \\
\hline Number of chambers & $n_{c h}=6$ \\
\hline Number of vanes & $n_{\text {vane }}=10$ \\
\hline Prototype displacement & $D=1.75 \mathrm{l} / \mathrm{rev}$ \\
\hline $\begin{array}{l}\text { Maximum pressure } \\
\text { during tests }\end{array}$ & $p_{h}=51 \mathrm{bar}$ \\
\hline $\begin{array}{l}\text { Compensation pressure } \\
p_{c 2}\end{array}$ & $p_{c 2}=$ \\
\hline $\begin{array}{l}\text { Rotational speed during } \\
\text { tests }\end{array}$ & $10,16,22,27 \mathrm{bar}$ \\
\hline $\begin{array}{l}\text { Maximum output torque } \\
\text { during tests }\end{array}$ & $n=6 \mathrm{rev} / \mathrm{min}$ \\
\hline Input flow during tests & $T_{\text {out }}=950 \mathrm{Nm}$ \\
& $Q_{\text {in }}=$ \\
\hline
\end{tabular}

there are asperity contacts and therefore mechanical friction between SOI. This friction loss depends on the normal force between SOI which decreases when $p_{h}$ increases. After $T_{\text {loss,min }}$ the lubricating regime between SOI is EHL and the corresponding friction loss is due to oil shearing. Hence, the dominating torque loss variation is from the vane-stator and stop-rotor contacts, which increase with $p_{h}$. The measured $h_{M C A}$ values are in agreement with the proposed loss behavior. They increase with $p_{h}$ and the value of $p_{c 2}$ has a significant effect on $h_{M C A}$. The trend of the $h_{M C A}$ curves is that the gap pressure distribution between the SOI has to overcome the compensation pressures before the gap height starts to increase. The $h_{M C A}$ measurements are in $\mu m$ which implies some uncertainties due to rotor surface asperities and thickness variations of the same order of magnitude. E.g. the curve for $p_{c 2}=27 b a r$ is expected to be below the curve for $p_{c 2}=22 b a r$ and then the gap height does not start to increase inside the pressure range of the tests.

It is expected that $T_{\text {loss,min }}$ occurs when the minimum gap height is just above zero for the entire SOI. But due to uneven height distribution over the SOI, the gap height for the entire SOI is not zero or above zero, when $h_{M C A}$ starts to measure deflections above zero. This is also confirmed by the model put forward in section 4 .

\section{Torque Loss Modeling of the Prototype Motor}

The aim of this section is to set up a model of the torque loss as a function of the pressure levels.
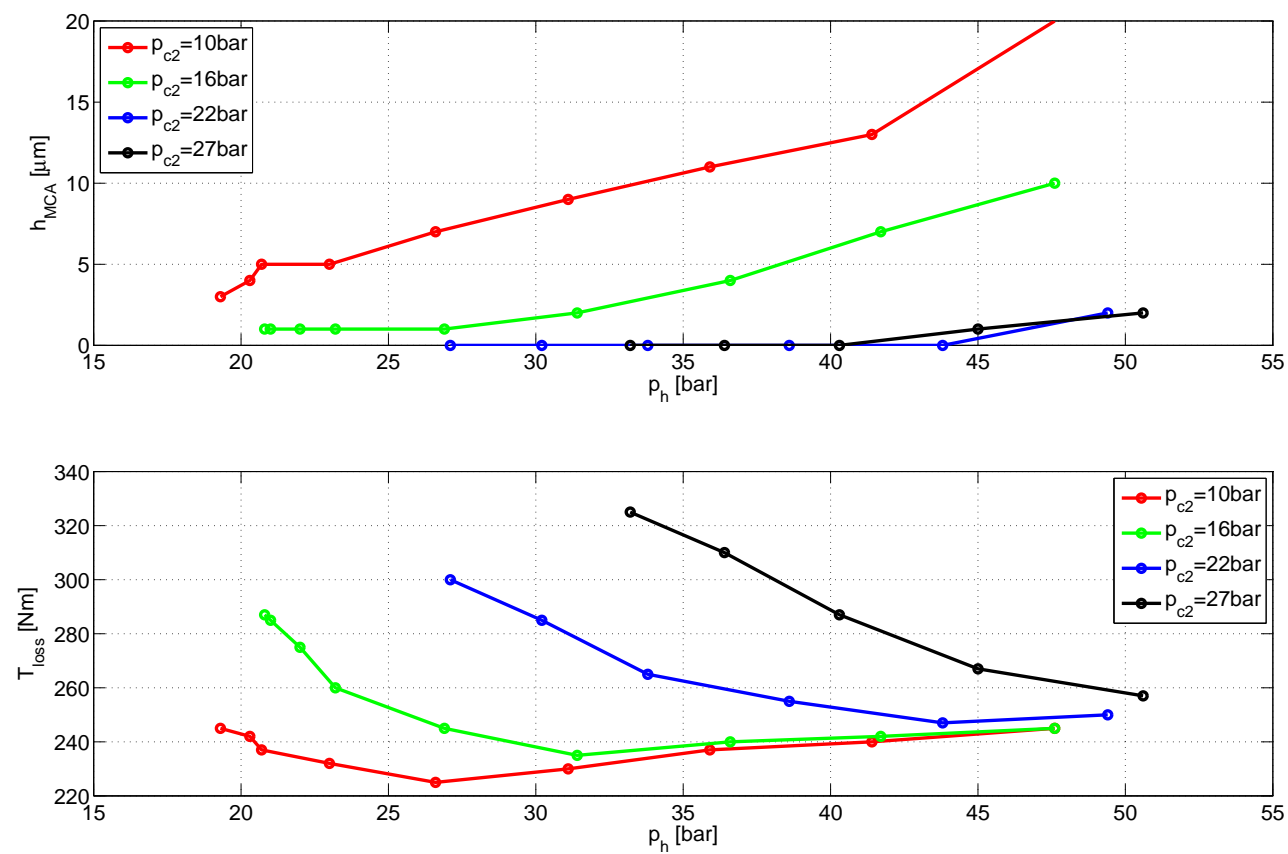

Figure 5: The measured deflections of the inner housing $h_{M C A}$ (top graph) and the measured torque losses $T_{l o s s}$ (bottom graph). Both are plotted with respect to the high pressure $p_{h}$. 


\subsection{Torque Loss of Hydraulic Motors}

The friction loss of hydraulic motors depends on the tribology of the surfaces moving relative to each other and it depends on the operational parameters of the motor. They are speed $n$, pressure $p$ and viscosity $\mu$, which are related to the total torque loss $T_{\text {loss }}$ as (Ivantysyn and Ivantysynova, 2003)

$$
\begin{aligned}
T_{\text {loss }}= & T_{\text {turb }}+T_{\text {visc }}+T_{\text {mech }}+T_{c s t} \\
= & C_{\text {turb }} \cdot \mu \cdot n^{2}+C_{\text {visc }} \cdot \mu \cdot \frac{n}{h} \\
& +C_{\text {mech }} \cdot \Delta p+C_{c s t},
\end{aligned}
$$

where $T_{\text {turb }}$ is torque loss due to friction in turbulent flow, $T_{v i s c}$ is due to viscous friction, $T_{\text {mech }}$ is due to mechanical friction and $T_{c s t}$ is constant torque loss mainly due to sealings. The gap height is denoted $h$.

Eqs. (2) and (3) describe the conceptual behaviour of the torque loss. The torque loss coefficients $C$ are those varying for different torque loss models and different motor principles. In the litterature these loss coefficients were first given as constants (Wilson, 1946). Later on it was shown (McCandlish and Dorey, 1984) that both linear and nonlinear loss coefficients give more accurate results over a wider range of operating conditions.

In this work the velocity and viscosity are held constant. Hence, eq. (3) is reduced to

$$
T_{\text {loss }}=C_{v i s c} \cdot \mu \cdot \frac{n}{h}+C_{m e c h} \cdot \Delta p+C_{1} .
$$

The term $C_{m e c h} \cdot \Delta p$ takes the friction force between vane-stator and stop-rotor into account and $C_{1}$ is the constant torque loss to a given velocity and viscosity. The $C_{v i s c} \cdot \mu \cdot n / h$ term describes the torque loss due to viscous friction between the SOI. The term relies on the SOI to remain separated and without any mechanical contact. But, according to the measurements, mechanical contact between SOI exists. The torque loss in these gaps highly depends on the gap height and, subsequently, the compensation pressures. Therefore, with constant velocity and temperature, the torque loss of the prototype motor is subdivided into

$$
\begin{aligned}
T_{\text {loss }} & =T_{c s t}+T_{\text {mech }}+T_{S O I} \\
& =K_{1}+K_{2} \cdot \Delta p+T_{S O I}
\end{aligned}
$$

where $K_{1}$ and $K_{2}$ are loss coefficients derived from measurements.

From the experiments it is evident that there is a transition in the lubricating regime between the SOI. Depending on the pressure state the lubricating regime will, in general, go from boundary lubrication (BL) via mixed lubrication (ML) and towards elastohydrodynamic lubrication (EHL). The Stribeck curve that

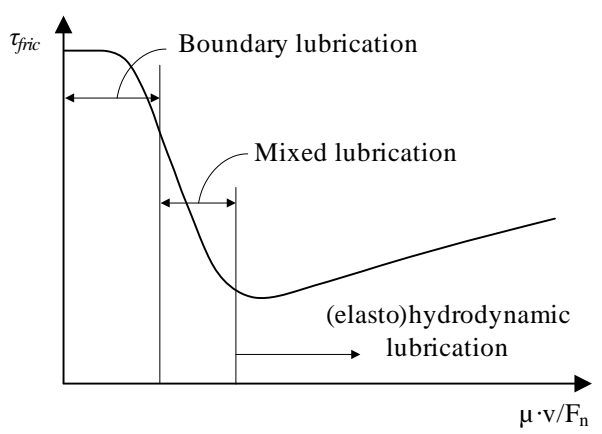

Figure 6: Stribeck diagram with lubrication regimes denoted.

relates the shear friction, $\tau_{\text {fric }}$, to the different friction regimes is illustrated in figure 6 . From the $T_{\text {loss }}$ and $h_{M C A}$ curves in section 3 it seems that the lubrication between the SOI is ML and at the beginning of EHL during the tests.

The friction in the diagram of figure 6 is based on viscosity $\mu$, velocity $v$ and the normal force $F_{n}$ between the gap surfaces (Bayer, 1994). The viscosity and velocity are constant during the tests. Hence, the friction loss variations only depend on normal force, according to figure 6 . But there is only a normal force between the SOI when the gap height is zero in some regions. Even though the gap height is above zero, there is potentially still asperity contact, and mechanical friction, due to the roughness of the surfaces. From that point of view, $F_{n}$ is not a valid parameter for the determination of the friction loss behavior in this case. Instead, the gap height distribution and the deflections of the surfaces are the parameters that influence the friction behaviour (Wang and Wang, 2006).

\subsection{Computation of Gap Height by Fluid Structure Interaction (FSI) Simulation}

The pressure distribution in the lubricating film between the SOI is influenced by the gap height which again influences the structural deflections. In this work the simultaneous computation of pressure and gap height is carried out by means of finite difference and finite element analyses, respectively, see also (Sørensen et al., 2012).

\subsubsection{Gap Pressure}

It is assumed that the rigid body motion of the rotor is only rotational and the density and viscosity of the oil is constant. Furthermore, the Reynolds equation is expressed in polar coordinates, because of the geometry and rotational motion of the rotor. Therefore the pressure distribution in the gaps between SOI is 


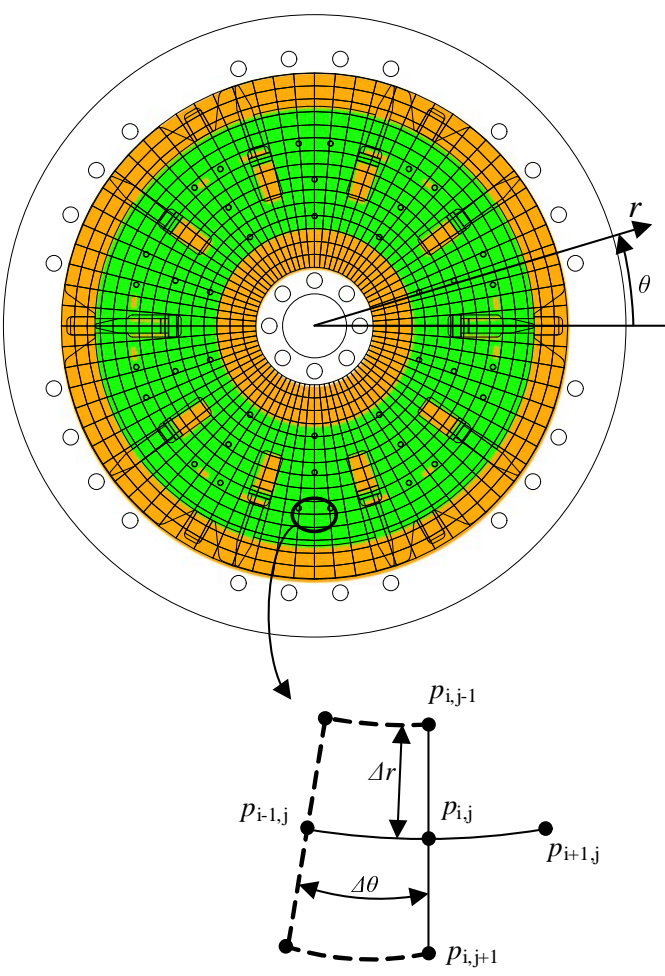

Figure 7: Finite difference mesh and boundary conditions. The color orange indicates that the pressure of the node is prescribed and green indicates that the pressure is to be determined.

(Beschorner et al., 2009)

$$
\begin{aligned}
\frac{\partial}{\partial r} \cdot\left(r \cdot h^{3} \cdot \frac{\partial p}{\partial r}\right)+\frac{1}{r} \cdot \frac{\partial}{\partial \theta} \cdot & \left(h^{3} \cdot \frac{\partial p}{\partial \theta}\right) \\
& =6 \cdot \eta \cdot r \cdot \omega \cdot \frac{\partial h}{\partial \theta} .
\end{aligned}
$$

Eq. (7) is solved numerically using the finite difference method for one rotor position that is representative for the mean pressure state over one motor revolution. By dividing the area into elements (see figure 7) and approximating the derivatives in eq. (7) by finite differences, $p_{i, j}$ is expressed by its surrounding pressures and can be determined by looping through the mesh until the pressure variation from previous iteration $\left(p_{i, j}^{\text {cur }}-p_{i, j}^{\text {prev }}\right)$ is smaller than a convergence tolerance.

\subsubsection{Gap Height}

The objective of the FEM model is to determine the structural deflections of the inner housing, which, with the end faces of the rotor, are forming the lubricating gaps of concern. The deflections of the rotor is assumed negligible because of the axial force balance. The input to the FEM simulations is the pressure distribution in the lubricating gap between the SOI and the vane actuation and compensation pressures. The output is the deflection of the face that forms the gap.

The pressures in the lubricating gap is added as nodal forces. The pressures from the fluid calculations are recalculated as nodal forces by

$$
F_{i, j}=p_{i, j} \cdot r_{i, j} \cdot \Delta r \cdot \Delta \theta
$$

The principle behind the FEM model is shown in figure 8. The stator and the inner and outer housing are glued together to one body with a gap between the inner and outer housing. The boundary condition is no displacement in the normal direction to the section cut of the stator. The areas with the applied forces are symbolized by bold red lines in figure 8 . The FEM model is meshed by three dimensional 8 node hexahedral elements.

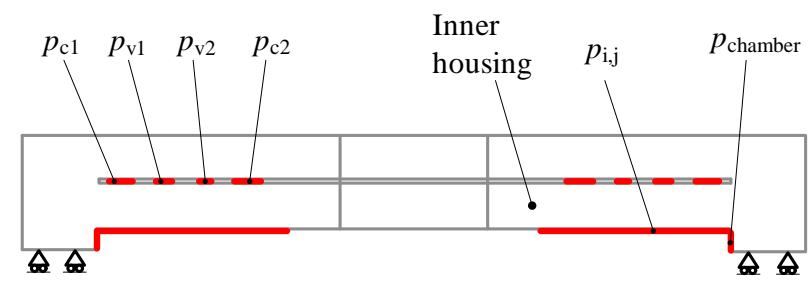

Figure 8: A section view of the FEM model with added pressures and boundary conditions.

\subsubsection{FSI Results}

The output from the FSI analysis is distribution of the gap height and the pressure between the SOI. The deflection of the inner housing that forms the gap and the pressure distribution in the gap to one test condition of the prototype motor are shown in figure 9. For the purpose of computational efficiency a linear FEM model was used in the FSI analysis, i.e., contact elements were not employed. The finite difference analysis is numerically limited in the way that it only converges as long as all gap heights are above a small threshold value, $h_{\epsilon} \approx 2 n m$. Hence, for any FSI analysis the inner housing and the rotor are given an initial position that ensures $h_{\min }^{(F S I)}>h_{\epsilon}$ for any load situations, see figure 10.

This gap height distribution is only valid for situations where the SOI are fully separated. In order to include situations where the SOI are partly separated or not separated at all, an offset gap height, $h_{o}$, is introduced. This offset value is subtracted from the FSI gap height to yield a nominal gap height, see also figure 


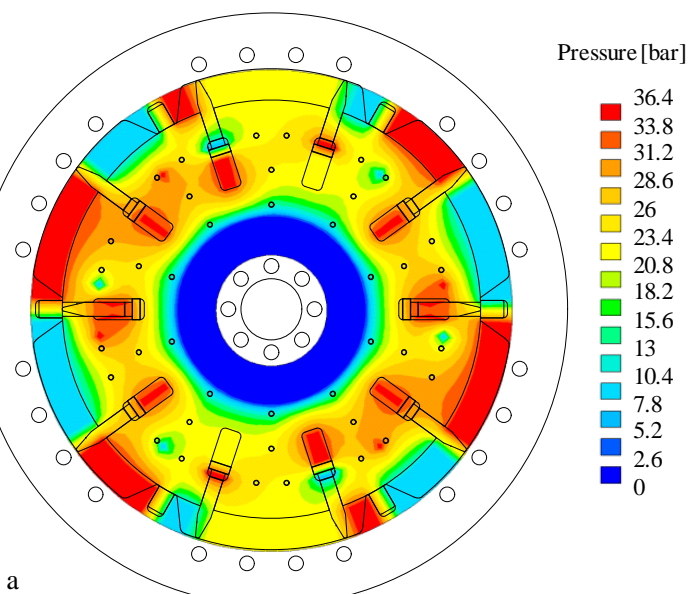

a

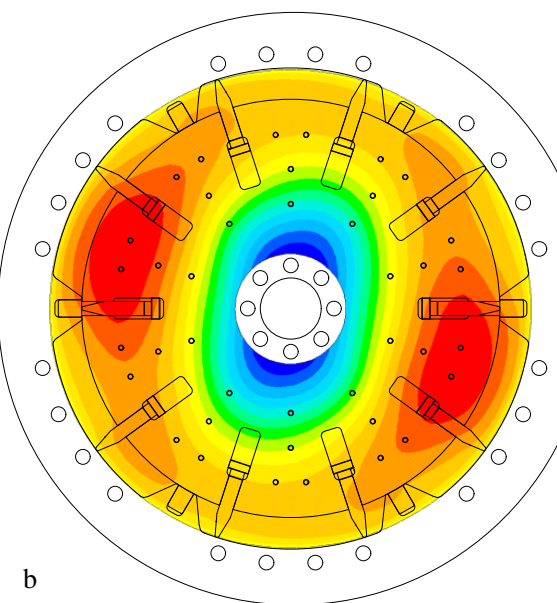

Deflection $[\mu \mathrm{m}]$

$\square .3$
$\square 3.79$
$\square .28$
$\square 2.77$
$\square 2.26$
$\square$
$\square .75$
$\square$
$\square .23$
$\square-72$
$\square-0.21$
$\square-0.3$
$\square-0.81$
$\square-1.32$
$\square-83$
$\square-2.35$
-2.86

Figure 9: The simulated pressure distribution (a) and the deflection of the inner housing (b) to one test condition of the prototype motor.
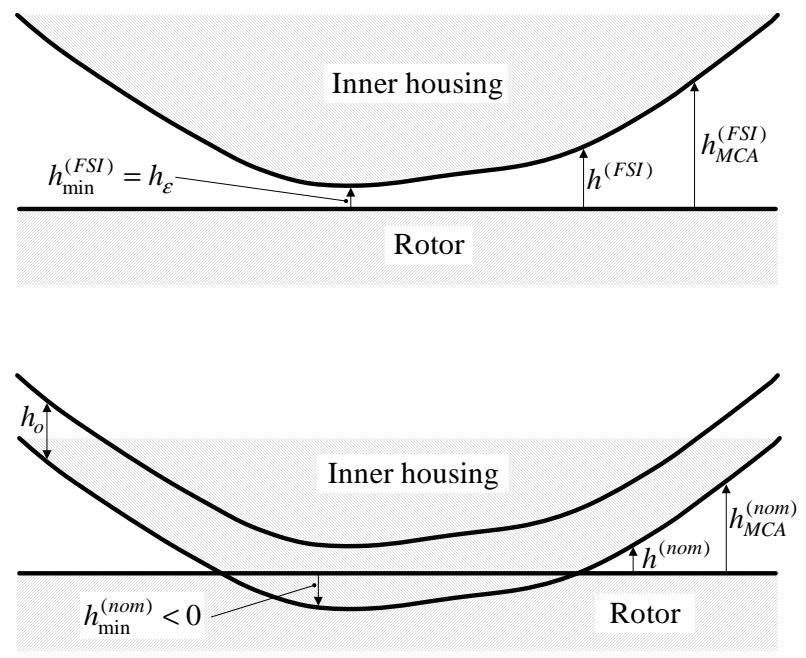

Figure 10: Illustration of $h^{(n o m)}, h^{(F S I)}$ and $h_{o}$.
10:

$$
h^{(n o m)}=h^{(F S I)}-h_{o}
$$

The offset gap height is identified by fitting the nominal gap height with the measured gap height at the MCA in situations where the SOI are fully separated yielding a value of $h_{o}=7.5 \mu \mathrm{m}$. The result of this is shown in figure 11 where a good accordance between gap increase vs. high pressure is observed for situations where the SOI are fully separated.

\subsection{Torque Loss based on Gap Height}

The total shear stress between the SOI, $\tau_{\text {fric }}$, in the ML regime is given by the sum of the shear stress of the asperity contacts, $\tau_{a s p}$, and the shearing of the oil, $\tau_{\text {fluid }}$, as (Gelinck and Schipper, 2000)

$$
\begin{aligned}
\tau_{\text {fric }} & =\tau_{\text {asp }}+\tau_{\text {fluid }} \\
& =\mu_{\text {fric }}\left(h^{\prime}\right) \cdot p^{\prime}+\mu \cdot \frac{v}{h^{\prime}},
\end{aligned}
$$

where $\mu_{\text {fric }}\left(h^{\prime}\right) \cdot p^{\prime}$ is the shear stress of the asperity contacts and $\mu \cdot v / h^{\prime}$ is the shear stress of the fluid. The friction coefficient function between the asperities, $\mu_{\text {fric }}$, is a function of the gap heights, given by

$$
\mu_{\text {fric }}\left(h^{\prime}\right)=K_{3} \cdot e^{-\frac{h^{\prime}-h_{t h r}}{h_{t h r}}}
$$

where $h_{t h r}$ is a treshold gap height that determines if mechanical contact. $p^{\prime}$ and $h^{\prime}$ are

$$
\begin{gathered}
h^{\prime}= \begin{cases}h_{t h r} & \text { if } h^{(n o m)}<h_{t h r} \\
h^{(\text {nom })} & \text { if } h^{(n o m)} \geq h_{t h r}\end{cases} \\
p^{\prime}= \begin{cases}p-K_{4} \cdot h^{(\text {nom })} & \text { if } h^{(n o m)}<h_{t h r} \\
p & \text { if } h^{(n o m)} \geq h_{t h r}\end{cases}
\end{gathered}
$$

By introducing $h^{\prime}$ singularities are avoided and a smooth transition from ML to EHL is ensured. Negative values of the nominal gap height correspond to a certain amount of indentation in the SOI. This is taken into account by means of the simple linear stiffness introduced in eq. (14) that is considered adequate to capture the increase in contact pressure.

Hence, the total simulated torque loss of the prototype motor, $T_{\text {loss }}^{(\operatorname{sim})}$, is

$$
\begin{aligned}
T_{\text {loss }}^{(\text {sim })} & =T_{c s t}^{(\text {sim })}+T_{m e c h}^{(\text {sim })}+T_{S O I}^{(\text {sim })} \\
& =K_{1}+K_{2} \cdot \Delta p+\tau_{f r i c} \cdot A \cdot r
\end{aligned}
$$

where $A$ is area and $r$ is radius. 


\section{Results and Comparison}

The coefficients in the torque loss expressions are determined based on the measurements.

\subsection{Parameter Identification}

\begin{tabular}{|l|c|}
\hline$K_{1}$ & $161 N m$ \\
\hline$K_{2}$ & $19.4 \cdot 10^{-6} \mathrm{~m}^{3}$ \\
\hline$K_{3}$ & $1.66 \cdot 10^{-3}$ \\
\hline$K_{4}$ & $2.38 \cdot 10^{12} \mathrm{~Pa} / \mathrm{m}$ \\
\hline$h_{t h r}$ & $1.06 \cdot 10^{-6} \mathrm{~m}$ \\
\hline
\end{tabular}

Table 2: Parameters derived from the tests.

The coefficients in the torque loss model are determined from the measurements by curve fitting as listed in table 2. The individual torque losses are shown in figure 12 . The constant term is very dominating.

\subsection{Comparison of Experimental and Simulated Torque Loss}

Both the experimental and simulated total torque losses are shown in figure 13 . The simulated total torque losses are determined by torque loss evaluations for every node on both side of the rotor. There is good correlation between the curves, which verify the friction model of section 4.3. The effect of $p_{c 2}$ on the hydromechanical efficiency when velocity and viscosity are constant is shown in figure 14 .

\section{Conclusions}

In this paper a model describing the torque losses in a hydraulic motor principle has been developed and ex-

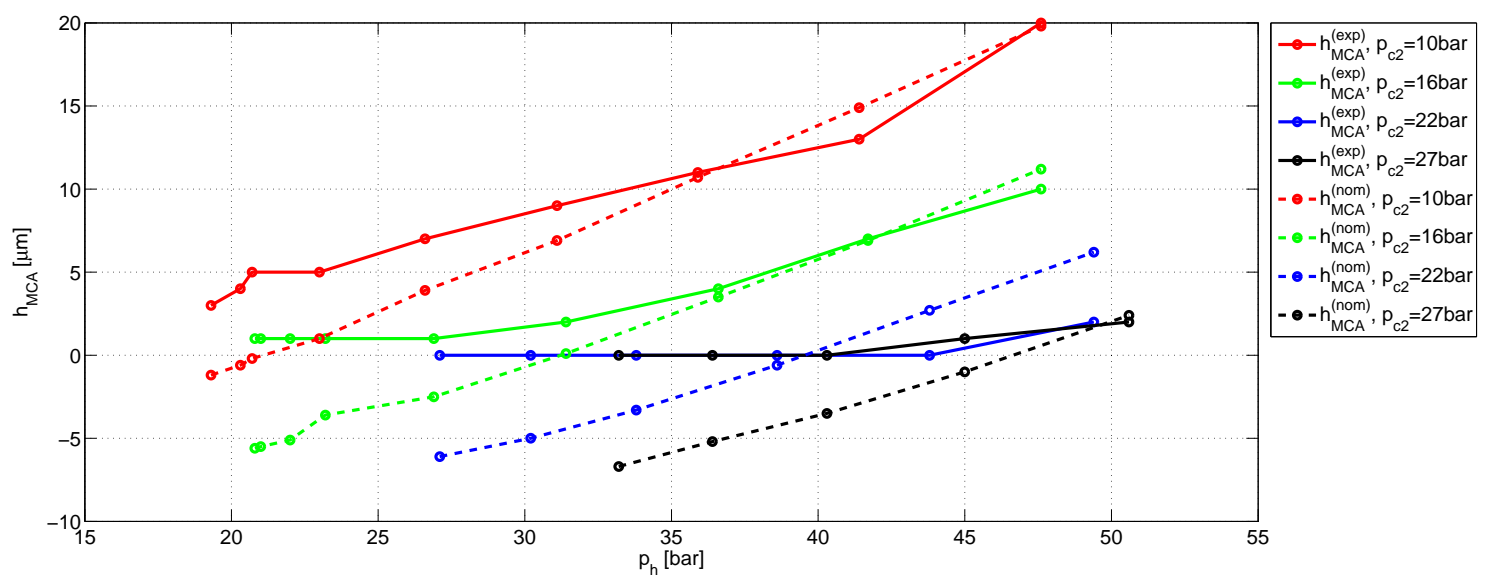

Figure 11: The simulated and experimental MCA deflections for the different $p_{c 2}$ values.

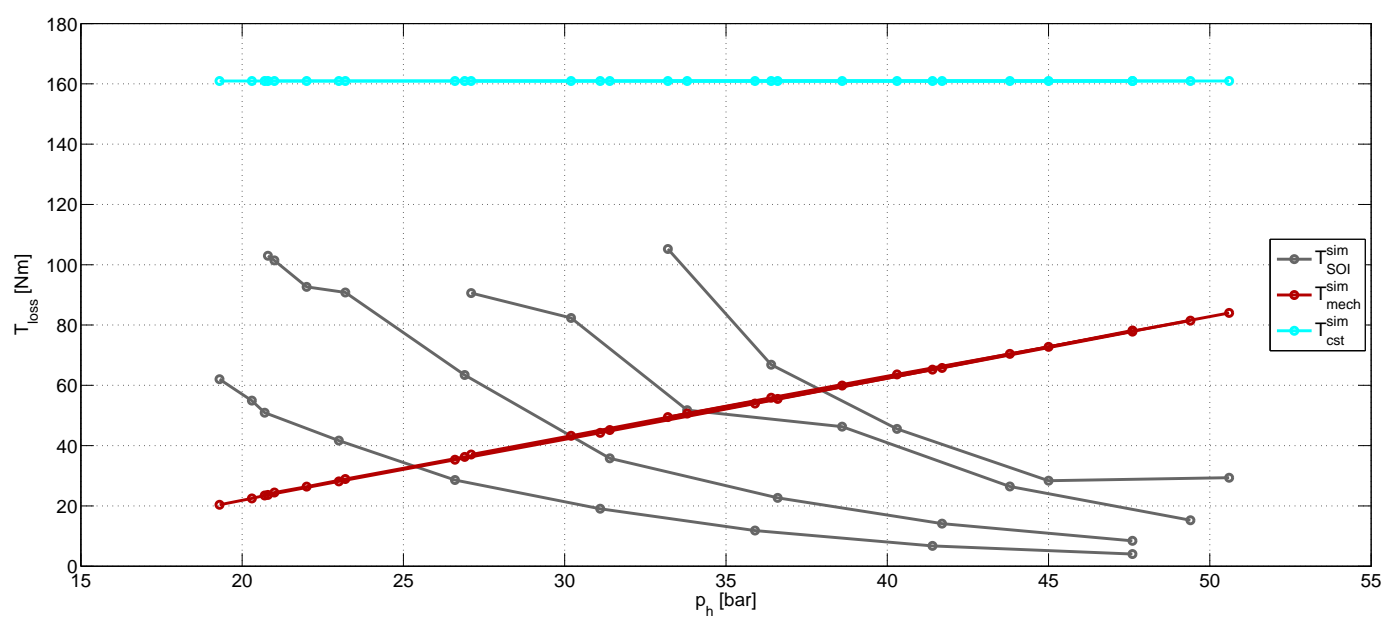

Figure 12: The individual simulated torque losses based on eq. (16) and after parameter identification. 


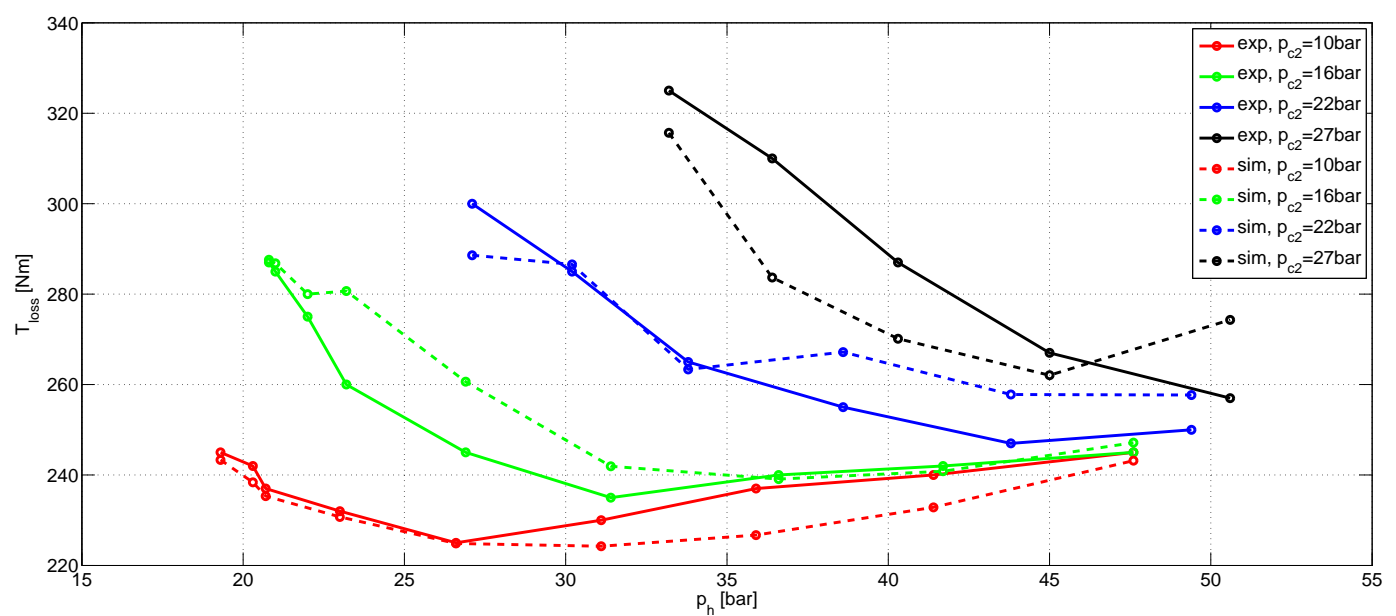

Figure 13: The simulated and experimental torque loss for the different $p_{c 2}$ values.

perimentally verified. The model describes the torque losses associated with mechanical and viscous friction under different lubricating conditions. The overall model is based on sub models of both the lubricating gap and the shear stress on the SOI. An FSI model is utilized for the computation of gap height and pressure distribution.

The most complex contribution to the hydromechanical loss is the friction loss between the SOI. The lubrication regime is either predominantly ML or predominantly EHL and the loss condition is highly dependent on the gap heights. In the FSI model there is mechanical contact if $h^{(n o m)}<h_{t h r}$. The behavior of the friction loss follows that of the Stribeck curve which only changes with the gap height in this study. The comparison of the simulated and measured hydromechanical loss shows a good correlation after the determination of the loss coefficients.

The correlation between the simulated and measured hydromechanical loss confirms the understanding of the interaction between the SOI. At the beginning of the $T_{\text {loss }}$ curves there are ML between the SOI with transition towards EHL when the total gap height is above zero.

The aim of the model development is a computational tool that can predict the effects and consequences of an up-scaling or modification of the motor principle, and, subsequently, minimizing the demands for prototyping. The potential for a further increase in specific displacement is increased when the motor is up-scaled. The aim is to up-scale the motor to displacements in the range of $200 \ldots 500 l / r e v$ in a very compact form, for extraordinary low speed high torque applications. Hence, minimization of structural deflec-

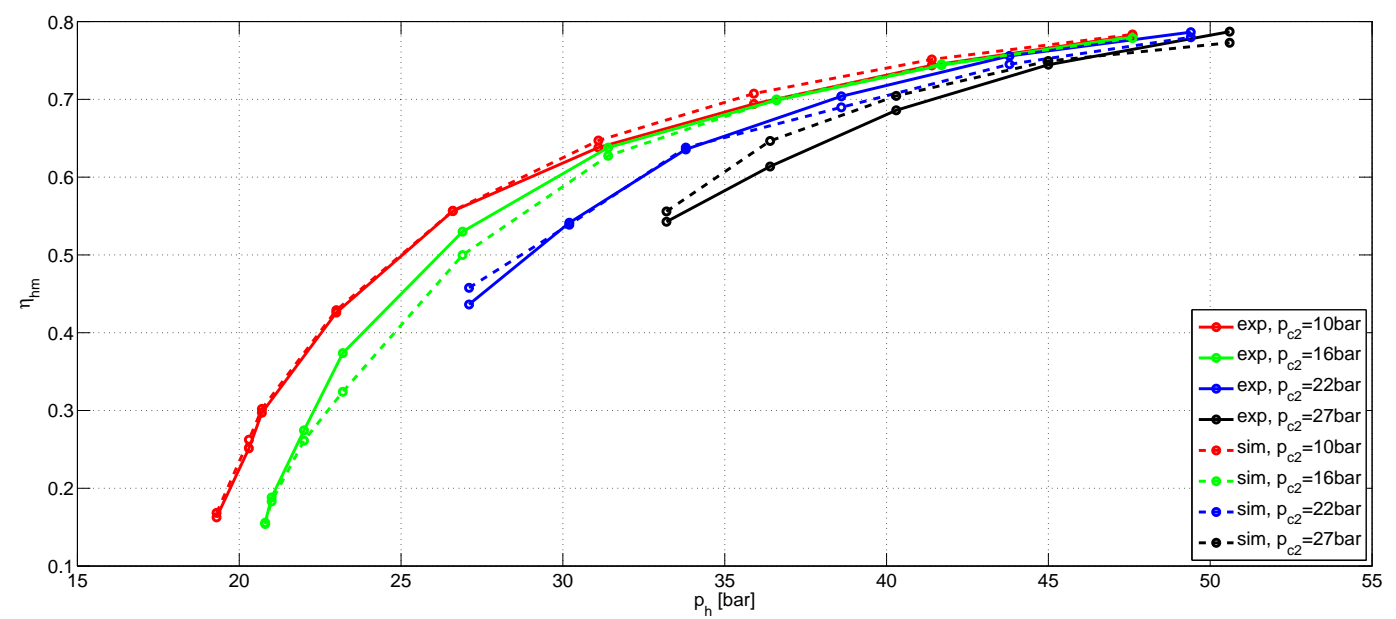

Figure 14: The simulated and experimental hydro-mechanical efficiency. 
tions are envisaged to be crucial in order to reach useful efficiencies over a wide range of operating conditions.

\section{References}

Bayer, R. Mechanical Wear Prediction and Prevention. Marcel Dekker, 1994.

Beschorner, K., Higgs, C., and Lovell, M. Solution of reynolds equation in polar coordinates applicable to nonsymmetric entrainment velocities. Journal of Tribology, 2009. 131.

Gelinck, E. and Schipper, D. Calculation of Stribeck curves for line contacts. Tribology International, 2000. 33:175-181. doi:10.1016/S0301679X(00)00024-4.

Ivantysyn, J. and Ivantysynova, M. Hydrostatic Pumps and Motors: Principles, Design Performance, Modelling, Analysis, Control and Testing. Tech Books International, 2003.

Ivantysynova, M. Prediction of pump and motor performance by computer simulation. First International Conference on Computational Methods in Fluid Power Technology, 2003.
McCandlish, D. and Dorey, R. The mathematical modelling of hydrostatic pumps and motors. Proc Instn Mech Engrs, 1984. 198B(10).

Murrenhoff, H., Piepenstock, U., and Kohmäscher, T. Analysing losses in hydrostatic drives. Proceedings of the 7th JFPS International Symposium on Fluid Power, 2008.

Sørensen, R., Hansen, M., and Mouritsen, O. Hydraulic yaw system for wind turbines with new compact hydraulic motor principle. EWEA 2011 Scientific Proceedings, 2011. pages 111-114.

Sørensen, R., Hansen, M., and Mouritsen, O. Numerical and experimental study of hydrostatic displacement machine. International Journal of Fluid Power, 2012. 13(2):29-40.

Wang, Y. and Wang, Q. Development of a set of Stribeck curves for conformal contacts of rough surfaces. Tribology Transactions, 2006. 49:526-535. doi:10.1080/10402000600846110.

Wilson, W. Rotary pump theory. Transaction of the ASME, 1946. pages 371-383. 